\title{
Initial-final mass relationship for stars of different metallicities
}

\author{
X. Meng ${ }^{1,2}$, X. Chen ${ }^{1}$, and Z. $\operatorname{Han}^{1}$ \\ 1 National Astronomical Observatories/Yunnan Observatory, the Chinese Academy of Sciences, Kunming, 650011, PR China \\ e-mail: conson859@msn.com \\ 2 Graduate School of the Chinese Academy of Sciences, PR China
}

Received 12 October 2007 / Accepted 19 May 2008

ABSTRACT

\begin{abstract}
Context. The initial-final mass relationship (IFMR) for stars is important in many astrophysical fields of study, such as the evolution of galaxies, the properties of type Ia supernovae (SNe Ia) and the components of dark matter in the Galaxy.

Aims. The purpose of this paper is to obtain the dependence of the IFMR on metallicity.

Methods. We assume that the envelope of an asymptotic giant branch (AGB) or a first giant branch (FGB) star is lost when the binding energy of the envelope is equal to zero $(\Delta W=0)$ and the core mass of the AGB star or the FGB star at the point $(\Delta W=0)$ is taken as the final mass. Using this assumption, we calculate the IFMRs for stars of different metallicities.

Results. We find that the IFMR depends strongly on the metallicity, i.e. $Z=0.0001,0.0003,0.001,0.004,0.01,0.02,0.03,0.04$, $0.05,0.06,0.08$ and 0.1 . From $Z=0.04$, the final mass of the stars with a given initial mass increases with increasing or decreasing metallicity. The difference in the final mass due to the metallicity may be up to $0.4 M_{\odot}$. A linear fit of the initial-final mass relationship in NGC 2099 (M 37) shows the effect of metallicity on the IFMR. The IFMR for stars of $Z=0.02$ obtained here matches well with those inferred observationally in the Galaxy. For $Z \geq 0.02$, helium WDs are obtained from the stars of $M_{\mathrm{i}} \leq 1.0 M_{\odot}$ and this result is supported by the discovery of numerous low-mass WDs in NGC 6791, which is a metal-rich old open cluster. Using the IFMR for stars of $Z=0.02$ obtained here, we have reproduced the mass distribution of DA WDs in Sloan DR4 except for some ultra-massive white dwarfs.

Conclusions. The trend that the mean mass of WDs decreases with effective temperature may originate from the increase of the initial metallicities of stars. We briefly discuss the potential effects of the IFMR on SNe Ia and at the same time, predict that metal-rich low-mass stars may become under-massive white dwarfs.
\end{abstract}

Key words. stars: white dwarfs - stars: supernovae: general

\section{Introduction}

White dwarfs (WDs) are the endpoint of the evolution of stars with initial masses ranging from about $0.1 M_{\odot}$ to about $8 M_{\odot}$. The vast majority of stars in the Galaxy belong to this mass range and over $97 \%$ of the stars in the Galaxy will eventually end up as WDs (Fontaine et al. 2001). As a result, WDs may give direct information about star formation during the Galaxy's earliest epochs (Gates et al. 2004). WDs are important in many fields. It has been about $50 \mathrm{yr}$ since Schmidt (1959) recognized the usefulness of WDs as cosmochronometers. WDs may also be the dominating component of dark matter in the Galaxy (Alcock et al. 1999; Chabrier 1999). WDs are very important for type Ia supernovae ( $\mathrm{SNe}$ Ia) since it is believed that $\mathrm{SNe}$ Ia form from the thermonuclear runaway of carbon-oxygen white dwarfs (CO WD) (see the reviews by Hillebrandt \& Niemeyer 2000; and Leibundgut 2000). It is well known that the masses of WDs are typically of the order of half that of the Sun, while their radii are similar to that of a planet. However, detailed knowledge of WDs is still lacking from observation and theory (Fontaine et al. 2001; Moroni \& Straniero 2002, 2007). Among all the uncertainties of WDs, the correlation between the initial-final mass relationship (IFMR) and metallicitiy is a very important one. It is well known that low metallicity leads to a larger CO WD for a given initial mass with $Z \leq 0.02$ (Umeda et al. 1999). However, it is necessary to check the cases of $Z>0.02$ since $Z \in[0.06-0.1]$ is possible for some ultra-luminous galaxies (Roberts \& Hynes 1994; Ruiz-Lapuente et al. 1995; Terlevich \& Forbes 2002).
The IFMR for stars over a large mass range (e.g. 0.8-8 $M_{\odot}$ ) is a powerful input to chemical evolution models of galaxies (including enrichment in the interstellar medium) and therefore can enhance our understanding of star formation efficiencies in these systems (Ferrario et al. 2005; Kalirai et al. 2007b). The IFMR is also an important input in modelling the luminosity functions of Galactic disk WDs and the cooling sequences of halo clusters, which may directly yield the age of the Galactic disk and halo components (Kalirai et al. 2007b). The IFMR represents the mass loss of a star over its entire evolution and it is possible to obtain some indications about the origin and evolution of hot gas in elliptical galaxies from the IFMR (Mathews 1990). Since Weidemann (1977) showed the first comparison between observations and theoretical predications of the IFMR, many observations especially during the last decade placed constraints on the relationship by studying the properties of white dwarfs in open clusters or field white dwarfs (Herwig 1995; Reid 1996; Koester \& Reimers 1996; Finley \& Koester 1997; Claver et al. 2001; Williams et al. 2004; Ferrario et al. 2005; Williams et al. 2006). Some empirical relations have also been suggested based on different observations (Weidemann 1983, 2000; Ferrario et al. 2005; Williams et al. 2006; Dobbie et al. 2006b). At the same time, numerous sets of stellar evolution models have been calculated to study the relationship in theory and to give the IFMRs for stars of different metallicity (Han et al. 1994; Girardi et al. 2000). Although much progress has been made in observation and theory, there still exist many uncertainties, i.e. what is the origin of the intrinsic scatter of WD mass or whether there is any 
dependence of the IFMR on the metallicity (Kalirai et al. 2005; Williams 2006). The correlation between the IFMR and the metallicity has been established in theory for many years (Han et al. 1994; Girardi et al. 2000), but the evidence of the dependence of the IFMR on the metallicity was not found until 2005 (Kalirai et al. 2005). Many theoretical calculations showed that the core mass at the first thermal pulse (TP) in the asymptotic giant branch (AGB) may be taken as the final mass (see the review by Weidemann 2000). However, these theoretical studies only focused on some special metallicities and it is also difficult for some stellar evolution codes to determine which is the first thermal pulse. In the paper, we use a simple but robust method to systemically study the IFMR over a wide metallicity range, i.e. $0.0001 \leq Z \leq 0.1$. We also briefly discuss the potential effect of the IFMR on SNe Ia.

In Sect. 2, we describe our model and physical inputs. We give the results in Sect. 3 and discussions and conclusions in Sect. 4.

\section{Model and physical inputs}

\subsection{The model}

When a star is in the AGB stage, its envelope may be blown off if the binding energy (BE) of the envelope changes from negative to positive (Paczyński \& Ziólkowski 1968). It is clearly shown in Fig. 1 of Han et al. (1994) that the BE of an AGB star changes from negative to positive through the AGB evolution (also Fig. 2 in this paper). The $\mathrm{BE}$ of the envelope can be calculated by

$\Delta W=\int_{M_{\mathrm{c}}}^{M_{\mathrm{s}}}\left(-\frac{G m}{r}+U\right) \mathrm{d} m$,

where $M_{\mathrm{c}}$ is the core mass, $M_{\mathrm{s}}$ is the surface value of the mass coordinate $m$, and $U$ is the internal energy of thermodynamics (including terms due to ionization of $\mathrm{H}$ and dissociation of $\mathrm{H}_{2}$, as well as the basic $\frac{3}{2} \mathfrak{R} T / \mu$ for a perfect gas). We assume that the envelope of a star is lost if $\Delta W=0$ and the core mass at this point is the final WD mass. The virtue of this method is that the mechanism of mass loss need not be considered. Han et al. (1994) used this assumption to obtain the IFMR for stars of $Z=0.02$ and $Z=0.001$. The binary population synthesis (BPS) incorporating their IFMRs well reproduced the mass distribution of planetary nebula nuclei (PNN). Their result indicated that this method is robust to calculate the final mass for a star of given initial mass and metallicity (see Han et al. 1994, for details).

In principle, the envelope has enough energy to escape to infinity when $\Delta W>0$. As mentioned in Han et al. (1994), however, it is not clear whether instant envelope ejection occurs or not. Radiation might take away some of the energy that is required to go into outward motion in order to achieve the envelope ejection, but it is possible that a series of oscillations on a dynamical time-scale with growing amplitude occur until $\Delta W$ is large enough to generate envelope ejection (Han et al. 1994). The discussion above means that $\Delta W \geq 0$ may only be a necessary condition to eject the envelope of an AGB star. Our assumption implies that a superwind starts at or after $\Delta W=0$. Fortunately, the change of the core mass during the superwind phase is negligible at low masses and relatively modest at high masses (Han et al. 1994).

To reducing computing time, we use the average evolution of thermally pulsing AGB models, i.e. skipping thermal pulses by taking a longer time-step. We discuss the influence of TPs and mass loss in Sect. 4.1.

\subsection{Physical inputs}

We use the stellar evolution code of Eggleton (1971, 1972, 1973), which has been updated with the latest input physics over the last three decades (Han et al. 1994; Pols et al. 1995, 1998). We set the ratio of mixing length to local pressure scale height, $\alpha=l / H_{\mathrm{p}}$, to 2.0 , and set the convective overshooting parameter, $\delta_{\text {OV }}$, to 0.12 (Pols et al. 1997; Schröder et al. 1997), which roughly corresponds to an overshooting length of $0.25 H_{\mathrm{P}}$. The range of metallicity is from 0.0001 to 0.1 , i.e. $0.0001,0.0003$, $0.001,0.004,0.01,0.02,0.03,0.04,0.05,0.06,0.08,0.1$. The opacity tables for these metallicties are compiled by Chen \& Tout (2007) from Iglesias \& Rogers (1996) and Alexander \& Ferguson (1994). For a given $Z$, the initial hydrogen mass fraction is assumed by

$X=0.76-3.0 Z$

(Pols et al. 1998), and then the helium mass fraction is $Y=$ $1-X-Z$. Based on the correlation between $X, Y$ and $Z$ used here, Pols et al. (1998) accurately reproduced the color-magnitude diagrams (CMD) of some clusters.

The mass loss might affect the IFMR. Han et al. (1994) have shown that the steady stellar wind based on the observational relation given by Judge \& Stencel (1991) will not significantly affect the IFMR. We also test the effect of Reimers's wind (1975) with $\eta=1 / 4$ on the IFMR of $Z=0.02$. A similar result to that in Han et al. (1994) is obtained. Buzzoni et al. (2006) also noted that Reimers wind parametrization poorly reproduces the relative number of planetary nebulae (PNe) in late-type galaxies, while a better fit of the relative number of PNe is obtained using the empirical IFMR in Weidemann (2000). This might imply that Reimers wind is an unreasonable mechanism to calculate the IFMR. We therefore do not include any wind mass loss in our calculation for any of the metallicities.

\section{Results}

\subsection{The initial-final mass relationship for stars of different metallicities}

Figure 1 shows the IFMRs for stars of different metallicities. The IFMRs in the paper may be approximated by two parabola segments, which is similar to that of Han et al. (1994) for $Z=0.02$ and $Z=0.001$. The fitted formulae of the IFMRs for stars of different metallicities are shown in Appendix A.

Han et al. (1994) showed that the BE of the envelope of Pop I low-mass stars $\left(M_{\mathrm{i}} \leq 1.0\right)$ may reach $\Delta W=0$ on the first giant branch (FGB) and helium WDs may be obtained. This phenomenon was used to explain the existence of low mass WD in Stein $2051 B$ and 40 EriB. We obtain a similar result to that of Han et al. (1994) for those stars with $Z \geq 0.02$ and $M_{\mathrm{i}} \leq 1.0 M_{\odot}$ (see those $M_{\mathrm{f}}<0.5 M_{\odot}$ in Fig. 1). The final masses of the He WDs for a given initial mass depend slightly on the metallicity. For a given initial mass, a larger He WD is obtained for a lower metallicity (see in Table 1). This is because for a perfect gas, the mean molecular weight, $\mu$, increases with metallicity according to Eq. (2) (Kippenhahn \& Weigert 1990) and the thermal energy therefore decreases with the metallicity. Although the final radii of the low-mass stars when $\Delta W=0$ in FGB slightly increases with the metallicity (see in Table 2), it does not significantly affect the gravitational energy of the envelope. So, the core mass dominating the gravitational energy of the envelope decreases with metallicity to counteract the decrease of thermal energy. 


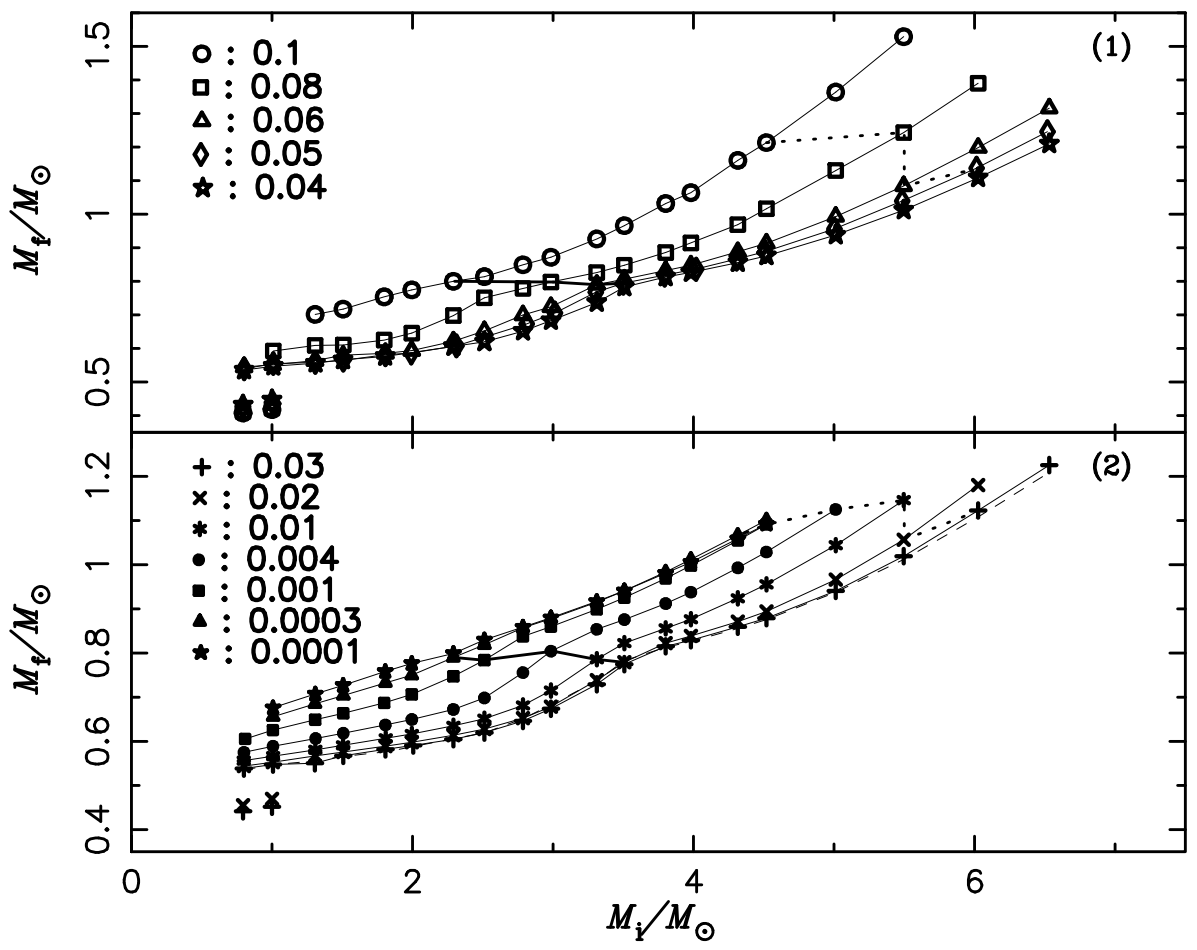

Fig. 1. Initial-final mass relationship (IFMR) for stars of different metallicities. The points of $M_{\mathrm{f}}<0.5 M_{\odot}$ represent helium WDs. The thick solid lines are the mass thresholds for the second dredge-up (see the text for details) and the thick dotted ones show the transition between carbon-oxygen white dwarfs and oxygen-neonmagnesium ones in our models. The dashed line in the lower panel shows the IFMR for stars of $Z=0.04$ as a comparison.

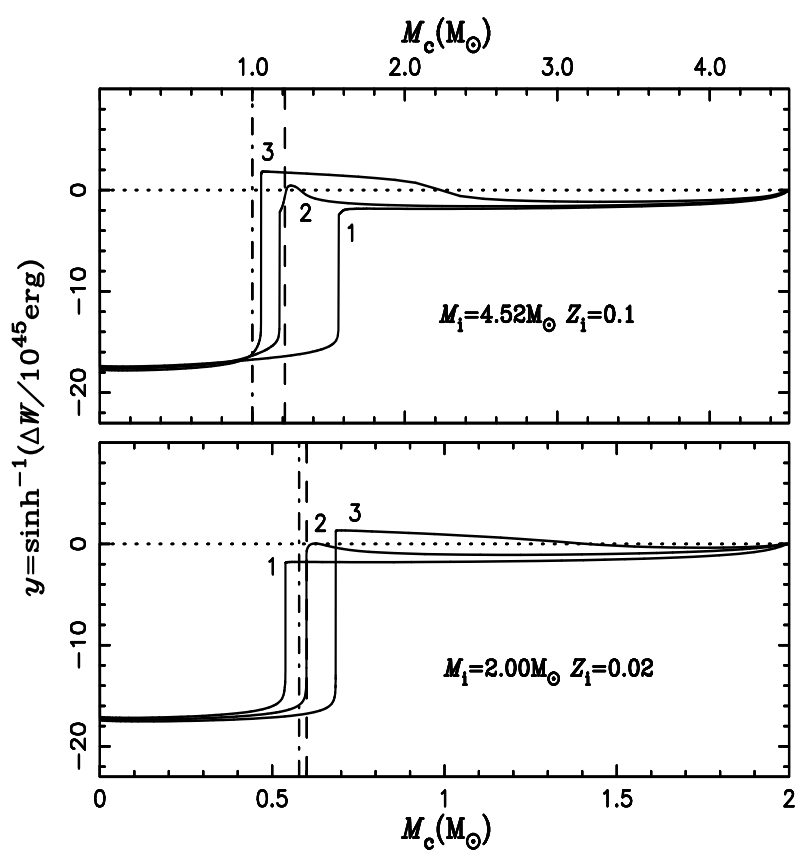

Fig. 2. The change of binding energy $\Delta W$ with the different choices of core mass, $M_{\mathrm{c}}$, (Eq. (1)) at three evolutionary stages for two AGB stars. Bottom panel is for $M_{\mathrm{i}}=2.00 M_{\odot}, Z_{\mathrm{i}}=0.02$; top panel is for $M_{\mathrm{i}}=4.52 M_{\odot}, Z_{\mathrm{i}}=0.1$. The numbers present the time sequence. The dependent variable, $y=\sinh ^{-1}\left(\Delta W / 10^{45} \mathrm{erg}\right)$, keeps the sign of $\Delta W / 10^{45} \mathrm{erg}$ and is a logarithmic function of $\Delta W / 10^{45} \mathrm{erg}$ when its absolute value is large, or a linear function when its absolute value is small. The dashed lines show the boundaries of the cores of the models defined in this paper, while the dot-dashed ones show the positions of helium burning shells.

According to the above finding, there are probably several under-massive WDs $\left(M_{\mathrm{WD}}<0.5 M_{\odot}\right)$ in metal-rich old clusters. Observationally, Kalirai et al. (2007a) recently found that most WDs are under-massive and the mean mass of the WDs is $\langle M\rangle=0.43 \pm 0.06 M_{\odot}$ in NGC 6791, which is one of the oldest $(8-12 \mathrm{Gyr})$ and most metal-rich $([\mathrm{Fe} / \mathrm{H}]=+0.4 \pm 0.1)$ open clusters in the Galaxy (Peterson \& Green 1998; Chaboyer et al. 1999; Stetson et al. 2003; Gratton et al. 2006; Origlia et al. 2006). According to the high metallicity and the old age of NGC 6791, the stars with $M_{\mathrm{i}} \sim 1.0 M_{\odot}$ in NGC 6791 likely have left the main sequence and moved into the FGB phase (the life of stars of $M_{\mathrm{i}}=1.0 M_{\odot}$ and $Z=0.04-0.06$ on the main sequence is about 7.5-9 Gyr, Chen \& Tout 2007). Kalirai et al. (2007a) suggested that the under-massive stars are helium WDs that have experienced enough mass loss on the FGB to avoid the helium flash, which naturally resolves the age discrepancy of the cluster within the white dwarf cooling theory $(2.4 \mathrm{Gyr})$ and from the main-sequence turnoff (8-12 Gyr) (Bedin et al. 2005).

It is obvious from Fig. 1 that there is a dependence of IFMR on the metallicity. The IFMR reaches its lower limit at $Z=0.04$, i.e. the final mass is smallest for $Z=0.04$ for a given initial mass. It is well known that for stars with $Z \leq 0.02$, the effect of decreasing metallicity is similar to that of increasing mass, i.e. the evolutionary track of a low-mass star with a low metallicity is similar to that of a larger one with a higher metallicty (Umeda et al. 1999). The result of Chen \& Tout (2007) reconfirmed this and suggested an opposite trend when $Z \geq 0.04$, i.e. for stars with a given initial mass, the age decreases with metallicity and the luminosity increases with metallicity (see Fig. 2 and Table 1 in that paper). That the effect of the metallicity on the evolution of a star is not monotonic is derived from the correlation between $X, Y$ and $Z$ used in this paper. The three components all contribute to opacity, which dominates the evolution of a star with a certain mass. As is well known, the opacity increases with $X$ or $Z$ and decreases with $Y$. The effect of increasing opacity is similar to that of decreasing mass. For $Z<0.04$, the change of $Z$ dominates the change of the opacity, while the changes of $Y$ and $X$ dominate the change of the opacity for $Z \geq 0.04$.

According to our calculation, the metallicities will result in a scatter of the final masses. For a certain mass, the scatter may be up to $0.4 M_{\odot}$, which depends on the differences of metallicity 
Table 1. The masses (in $M_{\odot}$ ) of helium WDs for different metallicities (in $Z_{\odot}$, Row 1) and different initial masses (Col. 1, in $M_{\odot}$ ).

\begin{tabular}{cccccccc}
\hline \hline$M_{\mathrm{i}}$ & 1.0 & 1.5 & 2.0 & 2.5 & 3.0 & 4.0 & 5.0 \\
\hline 0.79 & 0.4554 & 0.4415 & 0.4311 & 0.4257 & 0.4185 & 0.4108 & 0.4074 \\
1.00 & 0.4697 & 0.4518 & 0.4463 & 0.4354 & 0.4318 & 0.4213 & 0.4176 \\
\hline
\end{tabular}

Table 2. The final radius $\left(\log R_{\mathrm{f}} / R_{\odot}\right)$ when $\Delta W=0$ is on the first giant branch for different metallicities (in $Z_{\odot}$, Row 1) and different initial masses $\left(\right.$ Col. 1, in $\left.M_{\odot}\right)$.

\begin{tabular}{cccccccc}
\hline \hline$M_{\mathrm{i}}$ & 1.0 & 1.5 & 2.0 & 2.5 & 3.0 & 4.0 & 5.0 \\
\hline 0.79 & 2.2323 & 2.2419 & 2.2462 & 2.2523 & 2.2596 & 2.2688 & 2.2796 \\
1.00 & 2.2507 & 2.2531 & 2.2595 & 2.2610 & 2.2658 & 2.2734 & 2.2784 \\
\hline
\end{tabular}

and initial stellar mass. Williams (2006) compared the IFMR for stars in NGC 2099 (M 37, $Z=0.01$, Kalirai et al. 2005) with that in other two clusters (Hyades \& Praesepe, $Z=0.02$, Perryman et al. 1998; Claver et al. 2001) and claimed that "any metallicity dependence of the IFMR for $M_{\mathrm{i}} \approx 3 M_{\odot}$ must be smaller than $\Delta M_{\mathrm{f}} \approx 0.05 M_{\odot}$ ". The difference of the final mass for $M_{\mathrm{i}} \approx 3 M_{\odot}$ between $Z=0.02$ and $Z=0.01$ in this paper is about $0.04 M_{\odot}$, which is consistent with the result of Williams (2006).

We assume that the remnant is a CO WD if carbon and oxygen have not been ignited in a star when $\Delta W=0$ (see the thick dotted lines in Fig. 1). In Table 3, we show the maximum masses of the CO WDs, $M_{\max }^{\mathrm{CO}}$, for different metallicities. Note that the values of the $M_{\max }^{\mathrm{CO}}$ probably have some differences from the real ones because of the grid density of the models in this paper. In our study, the $M_{\max }^{\mathrm{CO}}$ are $1.2431 M_{\odot}$ and $1.2132 M_{\odot}$ for $Z=0.08$ and $Z=0.1$, respectively. Generally, the mass of a CO WD is less than $1.20 M_{\odot}$, otherwise carbon will be ignited in the region with the highest temperature and the final remnant after the ejection of its envelope is an oxygen-neon-magnesium $(\mathrm{ONeMg})$ WD. The overestimated values in our models for $Z=0.08$ and $Z=0.1$ mainly come from the determination of the core mass $M_{\mathrm{c}}$. As discussed by Han et al. (1994), it is difficult to determine where the boundary of the core lies. We chose different core masses $M_{\mathrm{c}}$ from the center to the surface and calculated the BE $\Delta W$ as a function of $M_{\mathrm{c}}$. Three evolutionary stages, i.e. before, at and after $\Delta W=0$, are selected to check the evolution of $\Delta W=0$ for two AGB stars. The results are shown in Fig. 2. In the figure, we see that there are three portions: (i) an inner region where $\Delta W$ increases slowly; (ii) a zone where $\Delta W$ increases sharply, the zone including the H-burning shell; and (iii) an outer portion where $\Delta W$ varies slowly with $M_{\mathrm{c}}$. We take the value near but outside the transition between (ii) and (iii) as $M_{\mathrm{c}}$ (the dashed lines in the figure) to be sure that $\Delta W$ is not very sensitive to $M_{\mathrm{c}}$. This choice is good enough for stars with a thin transition between (ii) and (iii), i.e. both $M_{\mathrm{i}}$ and $Z$ are not very large, e.g. $M_{\mathrm{i}}=2.00 M_{\odot}$ and $Z=0.02$ in the figure. However, for those with large initial mass and high metallicity, the transition between (ii) and (iii) is thick (see the model of $M_{\mathrm{i}}=4.52 M_{\odot}, Z_{\mathrm{i}}=0.1$ in Fig. 2) and it is difficult to choose the boundary of the core. This may result in an uncertainty in the final mass of $0.03 M_{\odot}$ for the model of $M_{\mathrm{i}}=4.52 M_{\odot}, Z_{\mathrm{i}}=0.1$. For most of the models in the paper, our choice of $M_{\mathrm{c}}$ does not significantly affect the results. The maximum uncertainty of the final masses derived from the choice of $M_{\mathrm{c}}$ is about $0.04 M_{\odot}$, which is from the model with $M_{\mathrm{i}}=5.50 M_{\odot}$ and $Z_{\mathrm{i}}=0.1$.

The value of $M_{\max }^{\mathrm{CO}}$ might also be overestimated if the star reaches $\Delta W=0$ again after the ejection of the H-rich envelope.
Table 3. The maximum masses (Row 3) of CO WDs for different metallicities (Row 1). The initial masses to get the final maximum CO WD are shown in Row 2.

\begin{tabular}{ccccccc}
\hline \hline$Z / Z_{\odot}$ & 0.005 & 0.015 & 0.05 & 0.2 & 0.5 & 1.0 \\
\hline$M_{\mathrm{i}} / M_{\odot}$ & 4.52 & 4.32 & 4.52 & 5.01 & 5.50 & 5.50 \\
$M_{\max }^{\mathrm{CO}} / M_{\odot}$ & 1.0918 & 1.0667 & 1.0912 & 1.1251 & 1.1461 & 1.0566 \\
\hline$Z / Z_{\odot}$ & 1.5 & 2.0 & 2.5 & 3.0 & 4.0 & 5.0 \\
\hline$M_{\mathrm{i}} / M_{\odot}$ & 6.03 & 6.03 & 6.03 & 5.50 & 5.50 & 4.52 \\
$M_{\max }^{\mathrm{CO}} / M_{\odot}$ & 1.1228 & 1.1081 & 1.1370 & 1.0842 & 1.2431 & 1.2132 \\
\hline
\end{tabular}

There is still a helium envelope around the CO core after a star loses its hydrogen-rich envelope and the helium envelope is thicker with increasing $M_{\mathrm{i}}$ and $Z$. For example, the mass of the helium envelope after the loss of the hydrogen-rich envelope may be as large as $0.2 M_{\odot}$ for a star of $M_{\mathrm{i}}=4.52 M_{\odot}$ with $Z=0.1$ (in Fig. 2, the dot-dashed lines show the positions of the helium burning shells). Then, the star becomes a helium red giant. With the expansion of the helium envelope, the envelope might reach $\Delta W=0$ and a part of the envelope might be lost again, which may lead to an overestimation of $M_{\max }^{\mathrm{CO}}$ (Han et al. 1994).

In Fig. 2, we see that, although the shape of $\Delta W$ with $M_{\mathrm{c}}$ has not changed during the AGB phase, the time sequence is different for the two stars, i.e. the region of the sharp increase of $\Delta W$ moves outward with time for the low-mass star, while it is opposite for the high-mass one. This difference comes from whether the second dredge-up occurs in a star or not. Generally, for Pop I stars $(Z=0.02)$, the second dredge-up occurs in earlyAGB (EAGB) stars defined by Iben (1983) if $M_{\mathrm{i}}>3.5 M_{\odot}$ (Busso et al. 1999), which reduces the mass of the H-exhausted core (see Fig. 6 of Meng et al. 2006). $\Delta W$ reaches zero during the process of core decrease as shown in the upper panel of Fig. 2. For the stars with $M_{\mathrm{i}}<3.5 M_{\odot}$, the second dredge-up does not occur and the core mass always increases with time as shown in the bottom panel of Fig. 2. When a star has a mass around $3.5 M_{\odot}$, the core mass is almost constant with the expansion of its envelope until $\Delta W=0$ (see Fig. 6 of Meng et al. 2006). The mass threshold for the second dredge-up changes with metallicity $Z$. We presented the mass threshold from our models for various $Z$ in Table 4 (see the thick solid line in Fig. 1) $)^{1}$. From Table 4, we see that, since the stellar evolution is non-monotonic with $Z^{2}$, the mass threshold is also non-monotonic with $Z$.

We notice that the final mass of the star of $M_{\mathrm{i}}=5.5 M_{\odot}$ with $Z=0.1$ is greater than $1.5 M_{\odot}$. It is likely that this remnant is not a white dwarf. When the star first arrives at the point of $\Delta W=0$, carbon and oxygen have been ignited and the $\mathrm{CO}$ core will become an $\mathrm{ONeMg}$ core. As described in the paragraph above, the star will become a helium giant star after the ejection of its hydrogen-rich envelope. If the helium envelope cannot reach $\Delta W=0$, the mass of the $\mathrm{ONeMg}$ core might reach the Chandrasekhar mass limit after about 1000-10000 yr and the star may explode as a type Ib supernova. Otherwise, an $\mathrm{ONeMg}$ WD would be its final fate.

For the cases of extremely low and high metallicities, the situation of $\Delta W=0$ cannot be met at the low-mass end and we cannot obtain the final mass through AGB stars (see Fig. 1).

\footnotetext{
${ }^{1}$ Note that the mass threshold may have an uncertainty of $0.15 M_{\odot}$ because of the grid density of the models in this paper. The mass interval is about $0.2-0.3 M_{\odot}$ for our models.

2 The non-monotonic effect of metallicity on stellar evolution is from Eq. (1). Please see the fourth paragraph in Sect. 3.1.
} 
Table 4. Mass threshold (Row 2, in solar mass) for different metallicities (Row 1, in solar metallicity). If a star has an initial mass higher than the mass threshold for a certain metallicity, the second dredge-up occurs in the star when helium is exhausted in the center.

\begin{tabular}{ccccccc}
\hline \hline$Z / Z_{\odot}$ & 0.005 & 0.015 & 0.05 & 0.2 & 0.5 & 1.0 \\
$M_{\mathrm{i}} / M_{\odot}$ & 2.30 & 2.30 & 2.50 & 3.00 & 3.30 & 3.50 \\
\hline$Z / Z_{\odot}$ & 1.5 & 2.0 & 2.5 & 3.0 & 4.0 & 5.0 \\
$M_{\mathrm{i}} / M_{\odot}$ & 3.50 & 3.50 & 3.50 & 3.30 & 3.00 & 2.30 \\
\hline
\end{tabular}

This is because for the stars with these extreme condition, the final mass is too high with respect to the initial mass and the envelope is too thin. For example, the mass of the core through the AGB is greater than $0.6 M_{\odot}$ for a star of $M_{\mathrm{i}}=0.8 M_{\odot}$ with $Z=0.0001$, and $\Delta W=0$ is not obtained before Eggleton's stellar evolution code is no longer applicable. This may imply that if the low-mass stars with extremely low and high metallicities experienced the AGB stage and became CO WDs, they might have a different mechanism to lose their envelopes than those with middle metallicities.

\subsection{Hertzsprung-Russel diagram}

The final positions $(\Delta W=0)$ of the stars before envelope loss with the different metallicities in a Hertzsprung-Russel diagram (HRD) are shown in Fig. 3. The dotted line gives the division of the FGB and AGB stars (the upper populations are AGB stars, while the lower ones are FGB stars). The effective temperature strongly depends on the metallicity since all the stars are near the Hayashi line which is determined by metallicity.

\subsection{Comparison between observations and theoretical predictions}

A comparison between observations and our theoretical predictions about IFMRs is shown in Fig. 4. Some empirical relations are also presented in the figure. The cross represents the mean error of the observational data. The solid line is the IFMR for stars of $Z=0.02$ obtained in this paper. The dashed, dotdashed and thick dotted lines are the empirical relations given by Weidemann (2000), Ferrario et al. (2005) and Williams (2006), respectively. Our theoretical IFMR for stars of $Z=0.02$ is consistent with the observations and the empirical relations, especially for low mass stars. This is an expected result since almost all clusters observed are open clusters in the Galaxy and their metallicities are around 0.02 .

\subsection{Evidence for the dependence of the initial-final mass relationship on the metallicities}

The IFMR derived from NGC 2099 might be evidence of the dependence of the IFMR on the metallicity. Kalirai et al. (2005) noticed that half of their data points lie in a region of the IFMRs for stars of $Z=0.008$ and $Z=0.02$ given by Marigo (2001). They suggest that this is the first evidence of the effect of metallicity on the IFMR. We revisit the data in Kalirai et al. (2005). The result is shown in Fig. 5. The solid line and the dot-dashed line are the results for $Z=0.02$ and $Z=0.01$, respectively. The dashed line is the best-fit linear least squares fit from the data in Kalirai et al. (2005). For convenience, we set the slope of the fitted line at that of the empirical relation given by Williams (2006), which is consistent with our IFMR for stars of $Z=0.02$ (see the linear thick dotted line in Fig. 4). The method used here is similar to that used in calculating a mean value since the mean value is the intercept of a line whose slope is zero. When fitting the line, we use the errors of the data as weight. As seen in Fig. 5, the fitted line is more consistent with the line of $Z=0.01$ than that of $Z=0.02$. Compared to the empirical relation given by Williams (2006), the fitted line moves upward by $0.05 M_{\odot}$. Interestingly, the estimate of the $\Delta M_{\mathrm{f}}$ between the IFMR for stars of $Z=0.02$ and that of $Z=0.01$ by Williams (2006) is $0.05 M_{\odot}$. As shown in Sect. 3.3, the IFMR for stars of $Z=0.02$ in this paper is consistent with observations in the Galaxy. Then, although the observational error is large, we reconfirm the discovery of Kalirai et al. (2005) that the IFMR derived from observations in NGC 2099 is possible evidence of the dependence of the IFMR on the metallicities and that the metallicity of NGC 2099 may be about 0.01 . Note that the observed metallicity of NGC 2099 is $[\mathrm{Fe} / \mathrm{H}] \sim-0.1$, which is less than the solar metallicity (Kalirai et al. 2005).

Another source of potential evidence is the Hyades cluster. The metallicity of the Hyades cluster $([\mathrm{Fe} / \mathrm{H}]=+0.17)$ is higher than that of NGC 2099, while its age is similar to that of NGC 2099 (Perryman et al. 1998; Kalirai et al. 2001, 2005). The mean mass of WD in NGC 2099 is $\langle M\rangle=0.80 \pm 0.03 M_{\odot}$ whereas WDs in Hyades have a mean mass of $\langle M\rangle=0.72 \pm$ $0.02 M_{\odot}$ (Claver et al. 2001; Kalirai et al. 2005). This also qualitatively matches with the trend obtained in this paper that for $Z<0.04$ - low metallicity, on average, leads to higher final mass.

Recently, Kalirai et al. (2007b) derived the first constraints on IFMRs at the low mass end. Their results are derived from three different clusters with different ages and metallicities, i.e. NGC 7789, NGC 6819 and NGC 6791. Since the ages and the initial masses for these clusters are different, their results cannot place a direct constraint on whether the IFMR depends on metallicity. However, our results are consistent with that of Kalirai et al. (2007b) within the errors. For example, WD7 in NGC $6791([\mathrm{Fe} / \mathrm{H}]=+0.4 \pm 0.1])$ has $M_{\mathrm{i}}=1.16_{-0.03}^{+0.04} M_{\odot}$ and $M_{\mathrm{f}}=0.53 \pm 0.02 M_{\odot}$, which is similar to our results of $Z=0.04$, i.e. $M_{\mathrm{f}}=0.5468 M_{\odot}$ for $M_{\mathrm{i}}=1.01 M_{\odot}$ and $M_{\mathrm{f}}=0.5560 M_{\odot}$ for $M_{\mathrm{i}}=1.31 M_{\odot}$. The cases of the other two clusters are similar. So, Kalirai et al. (2007b) might have provided indirect evidence of the dependence of the IFMRs on metallicity.

\subsection{The mass distribution of white dwarfs}

Incorporating the IFMR in this paper into Hurley's single stellar population synthesis code (Hurley et al. 2000, 2002), we simulate the distribution of the final mass. The results for age $\leq 15 \mathrm{Gyr}$ are shown in Fig. 6. For $Z=0.02$, a constant star formation rate of $3 M_{\odot} / \mathrm{yr}$ is assumed to match the observation in the Galaxy (Miller \& Scalo 1979; Timmes et al. 1997). For $Z=0.001$ and $Z=0.0001$, a single star burst is assumed. The star formation rate does not significantly affect the distribution of the final mass. Kepler et al. (2007) showed that the mass distribution of the single and non-magnetic DA stars in Data Release 4 of the Sloan Digital Sky Survey (hereafter Sloan DR4) can be fitted by four Gaussian fits. The Gaussian fits of the mass distribution of DA stars hotter than $T_{\text {eff }}=12000 \mathrm{~K}$ from Sloan DR4 are shown in Fig. 6 with dotted lines (Kepler et al. 2007). Incorporating the IFMR for stars of $Z=0.02$ into Hurley's rapid stellar evolution code, we reproduce the observational mass distribution of the WDs except for a small bump at $M_{\text {core }} \simeq 1.20 M_{\odot}$. The BPS results of Han (1998) showed that the final mass of the merger of two CO WD concentrates in $M_{\mathrm{f}} \simeq 1.20 M_{\odot}$ if the mass of the 


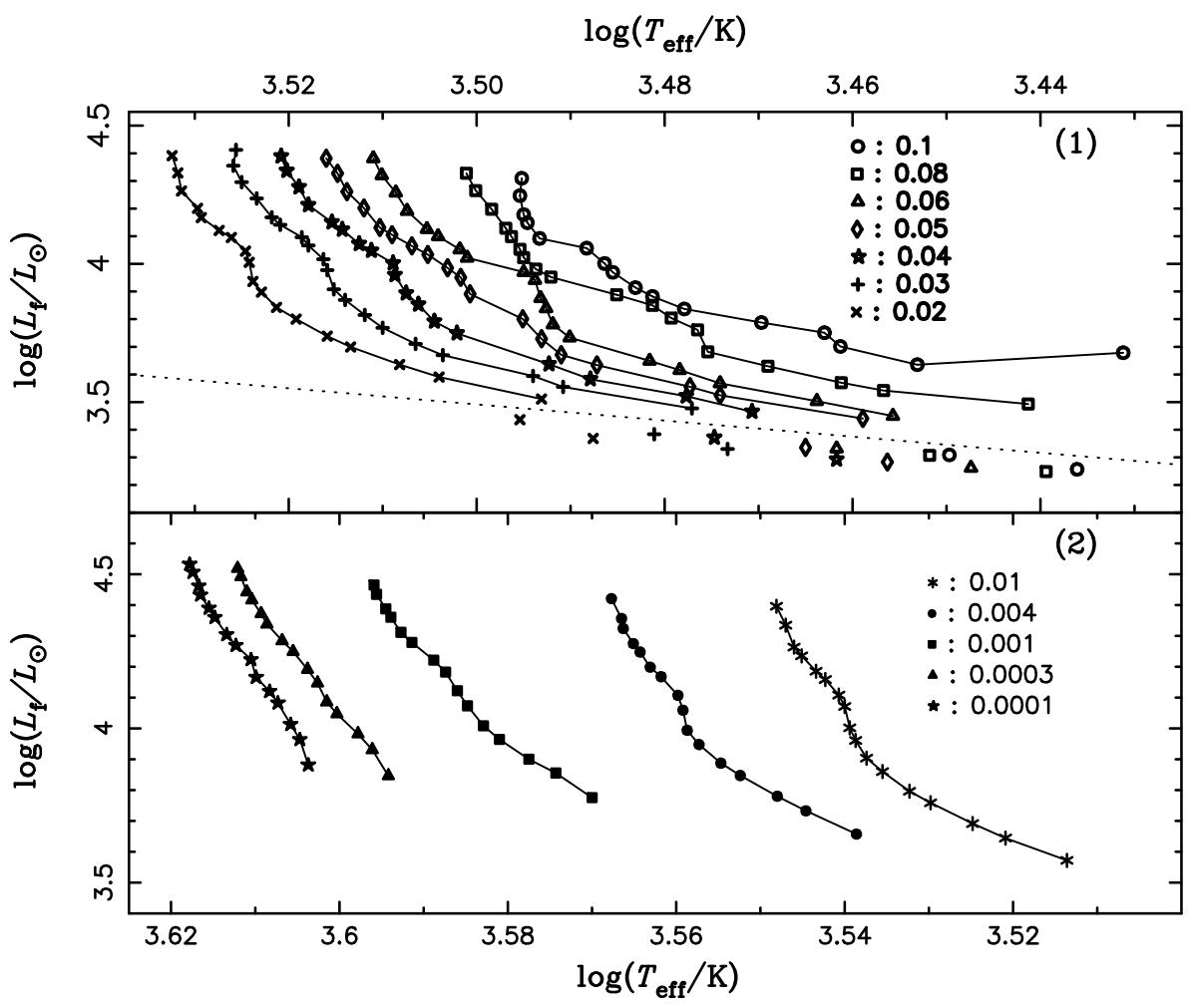

Fig. 3. The final positions $(\Delta W=0)$ of FGB/AGB in the Hertzsprung-Russel diagram (HRD) for different metallicities. In the upper panel, the final positions of AGB stars are above the dotted line, and the final positions of FGB stars are below. The upper panel shows models whose metallicity is high enough to permit the low-mass stars to reach $\Delta W=0$ on the FGB. Note that the scale of the abscissa in the upper panel is different from that in the lower panel.

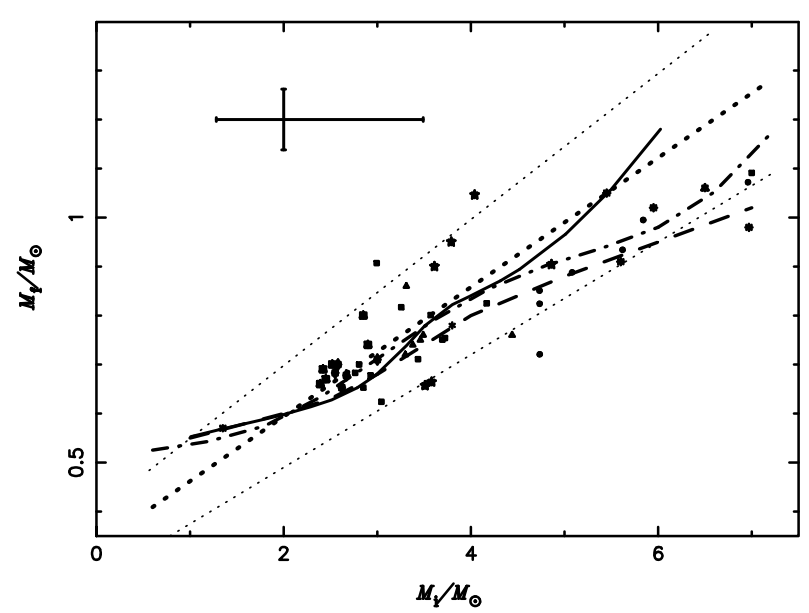

Fig. 4. Comparison between our theoretical prediction and observations. The cross represents the mean error of the observational value. Most of the observational data are from the observation of open clusters in the Galaxy and the data are from Reid (1996), Herwig (1995), Koester \& Reimers (1996), Finley \& Koester (1997), Claver et al. (2001), Williams et al. (2004), Ferrario et al.(2005) and Dobbie (2006b). The solid line is the IFMR for stars of $Z=0.02$ in the paper. The dashed, dot-dashed and thick dotted lines are the empirical relations given by Weidemann (2000), Ferrario et al. (2005) and Williams (2006), respectively. The two thin dotted lines show the range of error of the thick dotted line.

merger is less than the Chandrasekha mass limit (see in Sect. 4.2, for details). The bump in Sloan DR4 may be derived from the mergers of double degenerate systems.

In Sloan DR4, both DA WDs and DB WDs show a trend of mean mass decreases with effective temperature (Kepler et al. 2007). Kepler et al. (2007) suggested that this trend is not real and it would be derived from the absence of neutral particles in their model used to fit the WD spectral of Sloan DR4. From our

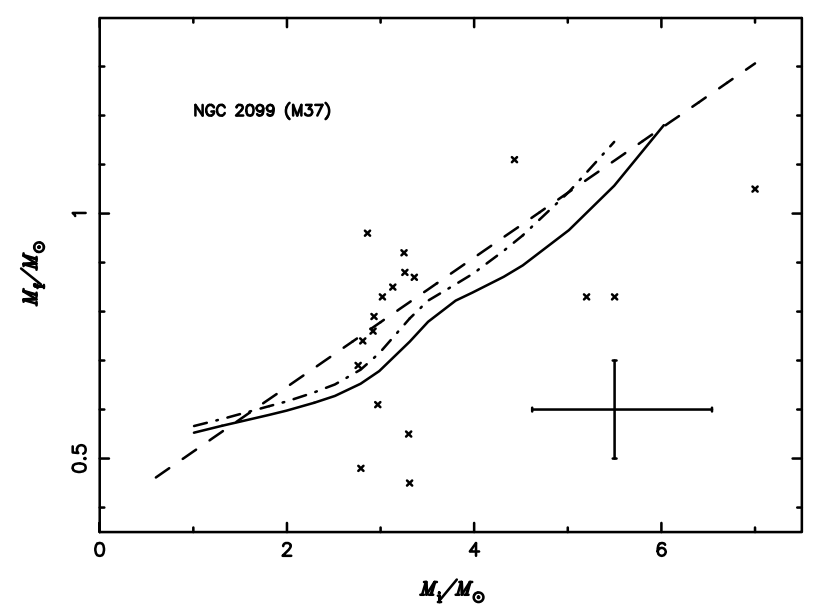

Fig. 5. Comparison between our results and the observation in NGC 2099 (M 37). The cross represents the mean error of observational data. The solid line and the dot-dashed one are the results found here for $Z=0.02$ and $Z=0.01$, respectively and the dashed line is the bestfit linear least squares fit of the observational data. The data are from Kalirai et al. (2005).

study, however, it is very likely that the trend is derived from the the effect of the metallicity as suggested by Wilson (2000). We know that stars with a low metallicity evolve faster than those with a high metallicity. So WDs from stars with a low metallicity form earlier and they have a longer time to cool. Meanwhile, WDs with a low metallicity are more massive for a certain initial mass if $Z<0.04$ (as shown in Figs. 1 and 6), and will cool more quickly (Kippenhahn \& Weigert 1990). Both of these facts imply that some massive WDs will be cooled and may not be observed. For the reasons above, we may expect a higher mean mass of WDs in globular clusters than that in open clusters or in the field. However, this prediction was not found to hold in NGC 6752, which is an old low-metallicity globular cluster 


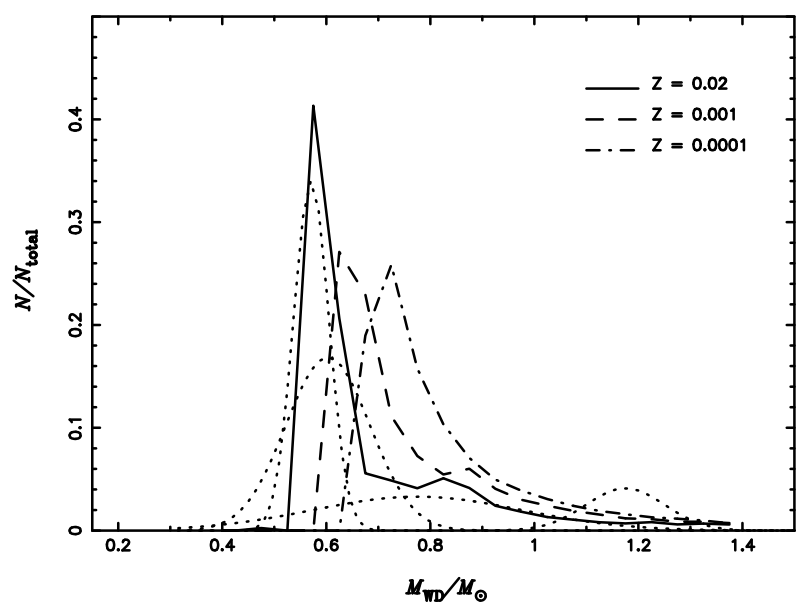

Fig. 6. Mass distribution of white dwarfs calculated by Hurley's single stellar population synthesis code incorporating our initial-final mass relationships for stars of different metallicities. The solid line represents the mass distribution of white dwarfs for $Z=0.02$, assuming a constant star formation rate of $3 M_{\odot} / y r$. Dashed and dot-dashed lines represent the mass distribution of white dwarfs for $Z=0.001$ and $Z=0.0001$ with a single star burst, respectively. The age at which WDs form in this diagram is less than 15 Gyr for all metallicities. The dotted lines are the Gaussian fits of the mass distribution of single and non-magnetic DA stars with $T_{\text {eff }} \geq 12000 \mathrm{~K}$ in Data Release 4 of the Sloan Digital Sky Survey (Kepler et al. 2007).

(Moehler et al. 2004). The reasons are as follows. Most globular clusters are very old and high mass WDs cool faster than low mass WDs (Kippenhahn \& Weigert 1990). So, the luminosity of high mass WDs are probably too low to be detected. We construct a model to examine the validity of the explanation. The basic parameters for the model are from Moehler et al. (2004), i.e. the distance modulus is set to $(m-M)=13$. $05-13$. 20 , which is used to deduce the mean value of the WD mass in the globular cluster $\left(0.53-0.59 M_{\odot}\right)$ by Moehler et al. (2004); the absolute magnitude of the Sun is 4 . 75 ; the limiting magnitude is set to $26^{\mathrm{m}}$ since the signal-to-noise ratio $(S / N)$ is low in Moehler et al. (2004) (see their Fig. 1). The lowest luminosity WDs that can be detected in NGC 6752 is $10^{-3.28}-10^{-3.22} L_{\odot}$, which means that a WD could not be detected in the cluster NGC 6752 if it cools for about 1 Gyr (Fontaine et al. 2001; Moroni \& Straniero 2002, 2007). The mass distributions of WDs with observational selection effects for different metallicities and different ages are shown in Fig. 7. The initial mass function (IMF) is set to $\phi(m) \propto m^{-2.35}$ (Salpeter 1955) and the ages of the progenitors of WDs are estimated by the life of the progenitors in the main sequence, multiplying by a factor of 1.1 (Hurley et al. 2000). The solid and dashed lines show the mass distribution of WDs for $Z=0.001$ and $Z=0.0001$. The age for each line is shown on the top of the line. It is evident that the position of the peak in the mass distribution of the WDs moves to a lower mass with time. If the age of a globular cluster with $Z=0.0001$ is higher than about $7 \mathrm{Gyr}$, the luminosity of all the WDs with $M_{\mathrm{i}}>1 M_{\odot}$ will be too low to be detected. NGC 6752 is a very old low-metallicity globular cluster (older than $12 \mathrm{Gyr}$ and $z=0.002$, Cassisi et al. 2000). For the case of $Z=0.001$, the peak mass of WDs is smaller than about $0.60 M_{\odot}$ at $12 \mathrm{Gyr}$, which is slightly larger than the observational value, $0.53 M_{\odot}-0.59 M_{\odot}$. Considering an observational error of $0.05 M_{\odot}$ and the real metallicity of NGC 6752, our results match the observations of Moehler et al. (2004). Since NGC 6752 is a globular cluster, the interaction among stars in the cluster might reduce the final mass.

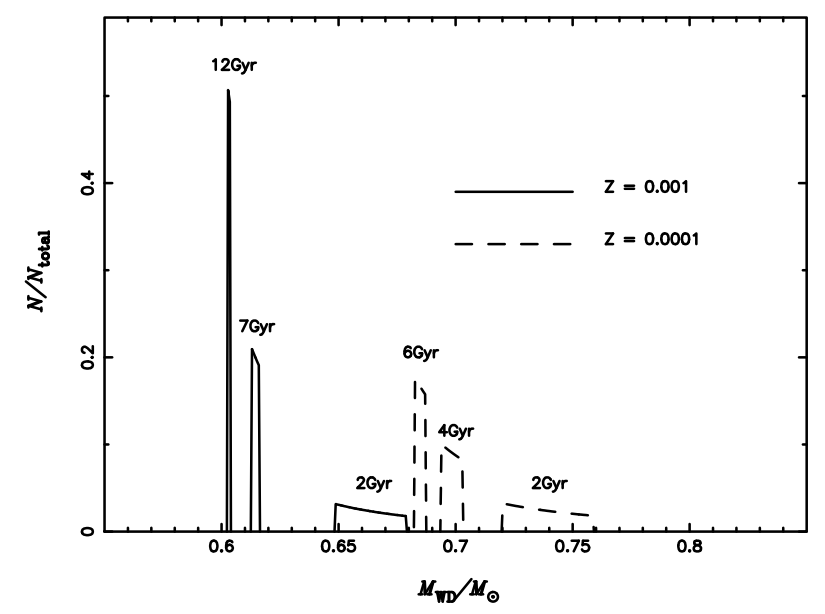

Fig. 7. Mass distribution of white dwarfs with observational selection effects. A single starburst is assumed for both metallicities. The age for each line is shown above the line.

The results of Fig. 7 indicate that the mass distributions of WDs are possibly good cosmochronometers, as first suggested by Schmidt (1959). On the other hand, the mass distribution of WDs also has the potential to distinguish the metallicitiy of a cluster since the mass peak with a given age is different for different metallicities. So, the mass distribution of WDs in globular clusters can help to break the degeneracy of the metallicity and the age if there are enough WDs to be observed.

\section{Discussions and conclusions}

\subsection{The uncertainty of the initial-final mass relationship}

The major uncertainty of the IFMR is from the assumption that the final mass is equal to the core mass when the binding energy of the envelope $\Delta W=0$ for an AGB/FGB star. Although the consistency between the mass distribution of WDs in this paper and that from Sloan DR4 supports this assumption, there is no direct observational evidence to verify it. Another widely accepted choice of the final mass is the core mass of a star at the beginning of the thermal-pulse AGB (TPAGB, the first TP or the end of early AGB, see the review by Weidemann 2000). From this choice, taking $Z=0.02$ as an example, the general trend of the IFMR is divided into three segments: (i) the core mass is almost constant from $1 M_{\odot}$ to $2.5 M_{\odot}$, around $0.52 M_{\odot}$, which is smaller than that in this paper by about $0.03-0.1 M_{\odot}$; (ii) the core mass strongly increases with the initial mass up to $4.0 M_{\odot}$. This trend is similar to that in this paper, but the core mass is less than that in this paper by $0.03-0.05 M_{\odot}$; (iii) the core mass slowly increases with the initial mass up to $6-7 M_{\odot}$. The final mass is lower than that in this paper by $0.1-0.2 M_{\odot}$. According to above discussion, the maximum variation of the final mass derived from different assumptions may be as large as $0.2 M_{\odot}$.

In this paper, we skipped the detailed evolution of TP and simply treated it as an average evolution, which may lose some information on the structural change of the envelope caused by a thermal pulse in the early phase of a thermally pulsing AGB. We therefore chose a model with $M_{\mathrm{i}}=3.00 M_{\odot}$ and $Z=0.02$ to examine the influence of thermal pulses on $\Delta W$ and the result is shown in Fig. 8. From the figure, we see that there is a time that $\Delta W$ changes its sign but returns immediately during a full amplitude of the TP. The change of the sign of $\Delta W$ results from the decrease of stellar radius, which results from the decrease of total luminosity (see panels 3 and 4). This implies that the 


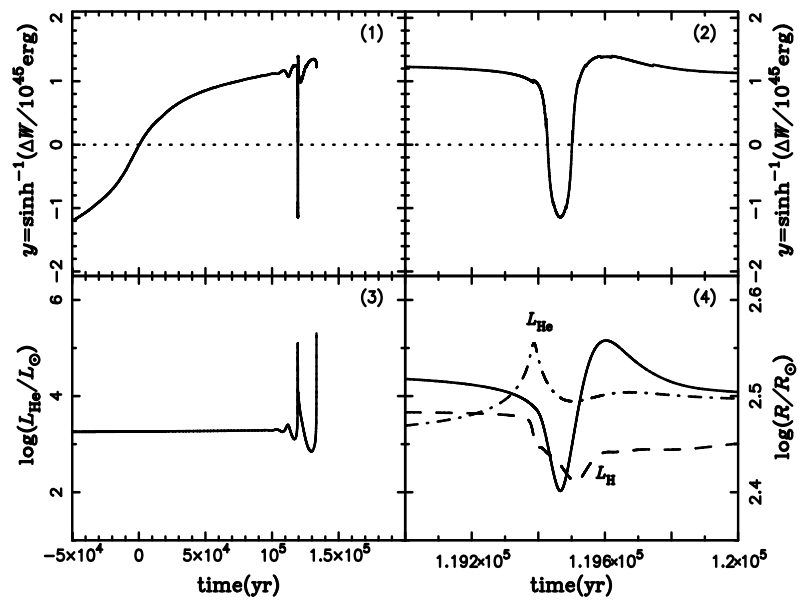

Fig. 8. Binding energy of the envelope (panels 1 and 2), helium luminosity (panel 3) and stellar radius (panel 4, solid line) as a function of time for a star with $M_{\mathrm{i}}=3.00 M_{\odot}$ and $Z=0.02$. The time when $\Delta W=0$ is set to be zero in the figure. Panels 2 and 4 are the enlarged images of panels 1 and 3 along abscissa. The dependent variable of $\Delta W$ in panels 1 and 2 is the same as that in Fig. 2. For comparison, helium and hydrogen luminosities are also plotted in panel 4 by dot-dashed and dashed lines, respectively, and their values are shown by the ordinate of panel 3.

superwind might be periodically interrupted, but the timescale of the interruption is very short $(\sim 100 \mathrm{yr})$, and thus its influence is small. There have been many studies focusing on the TPs and we briefly summarize them in the following. Generally, the final mass after several TPs is greater than the core mass at the first TP. Forestini \& Charbonnel (1997) calculated a model of $M_{\mathrm{i}}=3 M_{\odot}$ assuming a Reimers mass-loss law with $\eta$ increasing from 2.5 to 5.0 in the final AGB stage. They obtained $M_{\mathrm{f}}=0.60 M_{\odot}$ after 19 TPs, but the core mass is $0.54 M_{\odot}$ at the first TP. Dominguez (1999) showed that the thermal pulse starts when the core mass is $0.571 M_{\odot}$ for a star of $M_{\mathrm{i}}=3 M_{\odot}$, while the final mass increases to $M_{\mathrm{f}}=0.71 M_{\odot}$ after 26 TPs if the mass loss prescription of Groenewegen \& de Jong (1994) is adopted (Straniero et al. 1997). If the fairly strong mass-loss law of Blöcker (1995) was adopted, the final mass becomes $0.63 M_{\odot}$ after 20 TPs from $0.53 M_{\odot}$ at the first TP (Blöcker 1995). However, the final mass of $0.68 M_{\odot}$ is obtained from the model with $M_{\mathrm{i}}=3.0 M_{\odot}$ and $Z=0.02$ in this paper.

In Fig. 8, we see that $\Delta W=0$ for a star with $M_{\mathrm{i}}=3.00 M_{\odot}$ and $Z=0.02$ is achieved before the TPAGB phase, which might be very important for galactic chemical evolution since it is widely believed that s-process elements are created in the TPAGB phase of a low-mass star (Han et al. 1995; Busso et al. 1999). The $\Delta W=0$ for all the models in this paper is achieved at the EAGB phase. These results seem to indicate that s-process elements could not be created. In fact, as mentioned in Sect. 2.1, it is more likely that $\Delta W=0$ is only a lower time limit for the superwind, i.e. a superwind starts at or soon after $\Delta W=0$. Therefore, the stars achieving $\Delta W=0$ in the EAGB phase still have opportunities to enter the TPAGB phase, and contribute to s-process elements. So, the final mass shown in this paper might be different from the real one. As mentioned above, the core mass in $3 M_{\odot}$ models grows by about $0.1 M_{\odot}$ during TPAGB evolution. For a $1.5 M_{\odot}$ star with $Z=0.02$, the final core mass is about $0.03 M_{\odot}$ larger than that in this paper if the mass loss prescription of Groenewegen \& de Jong (1994) is adopted (Dominguez 1999). The main result in this paper then still holds.
In Fig. 4, we see that when $M_{\mathrm{i}}<3.5 M_{\odot}$, the IFMR in this paper is consistent with the empirical relations. However, for a higher initial mass, the IFMR in this paper is larger than the empirical relations given by Weidemann (2000) and Ferrario et al. (2005). This discrepancy is mainly from our assumptions. As mentioned in Sect. 2.1 and above paragraph, $\Delta W \geq 0$ is a necessary condition to eject the envelope of an AGB star. Since $\Delta W=0$ is achieved at the second dredge-up for a star with $M_{\mathrm{i}}>3.5 M_{\odot}$ during the EAGB phase and the core is decreasing at this phase, the necessary condition means that the final mass may be overestimated. The uncertainty of $M_{\mathrm{f}}$ attributed to the definition of the core in this paper may also reduce the discrepancy. Meanwhile, as mentioned in Sect. 3.1, the final mass might be overestimated if the helium envelope of a star reaches $\Delta W=0$ again after the ejection of the hydrogen-rich envelope. This overestimation is more likely for a high initial mass, since the helium envelope is thicker, and then reaches $\Delta W=0$ more easily (see Sect. 3.1). However, since the errors from the observations are also very large (see the error bar in Fig. 4), the IFMR obtained in this paper is located well in the error range and therefore is still consistent with observations.

\subsection{Low-mass white dwarf}

Kalirai et al. (2007a) suggested that helium white dwarfs may be found in metal-rich old clusters, i.e. NGC 6791. It is a reasonable assumption that the same mechanism in NGC 6791 would work for metal-rich field stars. Recently, Kilic et al. (2007c) reanalysed the data in Valenti \& Fischer (2005) and found that there have been metal-rich stars with $[\mathrm{Fe} / \mathrm{H}]>0$ at all times in the local Galactic disk, although the metallicity distribution of disk stars peaks below solar metallicity for stars with ages greater than about 5 Gyr. Considering that only $5 \%$ of all WDs in the local disk are single low-mass white dwarfs $\left(<0.45 M_{\odot}\right)$ (Liebert et al. 2005; Kepler et al. 2007) and the fraction of metal-rich stars with ages greater than 9 Gyr is $21 \%$, Kilic et al. (2007c) argued that only the stars of $[\mathrm{Fe} / \mathrm{H}]>+0.3$ can lose their hydrogen-rich envelope to produce helium WDs on the FGB. From our study, however, all stars with $M_{\mathrm{i}} \leq 1.0 M_{\odot}$ and $Z \geq 0.02$ will lose their hydrogen-rich envelope and finally become helium white dwarfs. This inconsistency may result from the overestimation of metal-rich stars in the sample of Valenti-Fischer (Reid et al. 2007) adopted by Kilic et al. (2007c)

As shown in our study, there should be numerous He WDs in metal-rich old clusters, which has indeed been observed in NGC 6791 (Kalirai et al. 2007a). Here, we emphasize that we do not assume any mass-loss mechanism while Kalirai et al. (2007a) assumed a metal-enhanced stellar wind on the red giant branch (RGB). Our study shows that $\Delta W=0$ in several FGB stars with high metallicities and low initial masses can be achieved before these stars reach the tip of the FGB, indicating that the relative number of the tip RGB stars in metal-rich old clusters is smaller than that in metal-poor clusters. This fact is directly observed in the RGB luminosity functions of two open clusters, e.g. NGC 188 and NGC 6791 (see Fig. 8 in Kalirai et al. 2007a). Meanwhile, a similar effect should be seen in the local population of RGB stars. Luck \& Heiter (see Fig. 9 in their paper, 2007) make a comparison of the metallicity histograms between dwarfs and giants within $15 \mathrm{pc}$ of the Sun and found that the dwarf population has a high metallicity tail extending to $[\mathrm{Fe} / \mathrm{H}] \sim 0.6$, while the giants show a sharp drop in numbers after $[\mathrm{Fe} / \mathrm{H}]=0.2$ and no giants with $[\mathrm{Fe} / \mathrm{H}]>0.45$ are observed in the field. 
The fate that low-mass metal-rich stars will become He WDs before helium is ignited on FGB might place constrains on planetary nebulae $(\mathrm{PNe})$. It is widely accepted that $\mathrm{PNe}$ originate from AGB stars or are related to binary evolution. For the case of binary evolution, one component in the binary system fills its Roche lobe at the AGB phase and the mass transfer is dynamically unstable, which leads to a common envelope phase. After the ejection of the common envelope, a PN may form. However, since some metal-rich stars may not experience an AGB phase, we might speculate that the number of PNe in metalrich galaxies is relatively lower than that in metal-poor galaxies. Interestingly, Buzzoni et al. (2006, see their Fig. 11) observed a relatively low number of $\mathrm{PNe}$ per unit galaxy luminosity in more metal-rich elliptical galaxies. Gesicki \& Zijlstra (2007) compared the mass distribution of the central stars of planetary nebulae (CSPN) with those of WDs. These two distribution are very different, i.e. the CSPN mass distribution is sharply peaked at $0.61 M_{\odot}$, ranging from $0.55 M_{\odot}$ to $0.66 M_{\odot}$, while the WD distribution peaks at a slightly lower mass and shows a much broader range of masses. Gesicki \& Zijlstra (2007) suggested that this difference may imply that only some WDs have gone through the PN phase. Our models provide a channel for WDs to avoid the PN phase.

Based on our results, WDs from single stars are always larger than $0.4 M_{\odot}$, whatever the composition of the WD. Then, extremely low-mass WDs $\left(0.2 M_{\odot}\right)$ are possible in binary systems or once in binary systems since no stellar population is old enough to produce such extremely low-mass WDs through single star evolution. Only recently, several extremely low-mass $\left(0.2 M_{\odot}\right)$ WDs were discovered in the field (Liebert et al. 2004; Kawka et al. 2006; Eisenstein et al. 2006; Kilic et al. 2007a), and some of them are companions of pulsars (van Kerkwijk et al. 2005). Kilic et al. (2007b) also found a low-mass WD in a binary system. If an extremely low-mass WD were a single star, it is very likely that it could have a very high space velocity since it may come from a close double-degenerate binary, where its companion has gone through a supernova event that disrupted the binary (Hansen 2003). LP 400-22 might be a case of this channel (Kawka et al. 2006). In any case, hard work is need to systematically search for the companions of WDs with mass less than $0.4 M_{\odot}$.

\subsection{The potential effect of the initial-final mass relationship on type la supernovae}

As the best cosmological distance indicators, type Ia supernovae (SNe Ia) have been successfully used to determine the cosmological parameters, e.g. $\Omega_{\mathrm{M}}$ and $\Omega_{\Lambda}$ (Riess et al. 1998; Perlmutter et al. 1999). The Phillips relation (Philips 1993) is used when taking SNe Ia as distance indicators. It is assumed that the Phillips relation is correct at high redshift, although the relation was obtained from a low-redshift sample. This assumption is precarious since the exact nature of $\mathrm{SNe}$ Ia is still unclear, especially the progenitor model and explosion mechanism (Hillebrandt \& Niemeyer 2000; Leibundgut 2000). If the properties of SNe Ia evolve with redshift, the results for cosmology might be different. Since metallicity decreases with redshift, a good way to study the correlation between the properties of SN Ia and redshift is to study the correlation between the properties of SN Ia and metallicity. It is widely believed that SNe Ia form from thermonuclear runaway of a carbon-oxygen white dwarf (CO WD) in a binary system. The CO WD accretes material from its companion to increase its mass. When its mass reaches its maximum stable mass, it explodes as thermonuclear runaway and almost half of the WD mass is converted into radioactive nickel-56 (Branch 2004). The mass of nickel-56 determines the maximum luminosity of SNe Ia. The higher the mass of nickel-56, the higher the maximum luminosity (Arnett 1982). Some numeric and synthetic results showed that metallicity may affect the final amount of nickel-56, and thus the maximum luminosity of SNe Ia (Timmes et al. 2003; Travaglio et al. 2005; Podsiadlowski et al. 2006). There is also much evidence about the correlation between the properties of SNe Ia and metallicity in observations (Branch \& Bergh 1993; Hamuy et al. 1996; Wang et al. 1997; Cappellaro et al. 1997), e.g. the maximum luminosity of SNE Ia is proportional to the metallicity (Shanks et al. 2002).

Two progenitor models of SNe Ia have competed for about three decades. One is a single degenerate model, which is widely accepted (Whelan \& Iben 1973). In this model, a CO WD increases its mass by accreting hydrogen- or heliumrich matter from its companion, and explodes when its mass approaches the Chandrasekhar mass limit. The companion may be a main-sequence star (WD + MS) or a red-giant star (WD + RG) (Yungelson et al. 1995; Li et al. 1997; Hachisu et al. 1999a, 1999b; Nomoto et al. 1999; Langer et al. 2000). Hachisu \& Kato $(2003 a, b)$ suggested that supersoft X-ray sources, which belong to the WD + MS channel, may be good candidates for the progenitors of SNe Ia. The discovery of the companion of Tycho's supernova also verified the reliability of the model (Ruiz-Lapuente et al. 2004; Ihara et al. 2007). Another progenitor model of SNe Ia is a double degenerate model (Iben \& Tutukov 1984; Webbink 1984), in which a system consisting of two CO WDs loses orbital angular momentum by gravitational wave radiation and merges. The merger may explode if the total mass of the system exceeds the Chandrasekhar mass limit (see the reviews by Hillebrandt \& Niemeyer 2000; and Leibundgut 2000). In both of the progenitor models, the CO WD which finally explodes to be a SN Ia should approach or exceed the Chandrasekhar mass limit. Obviously, a higher mass CO WD may fulfill this condition more easily than a low mass one. According to our results, the mass of a CO WD with a given initial mass will be higher in the circumstance with extremely high metallicity or extremely low metallicity than that in the middle-metallicity circumstance. Metallicity is therefore very relevant for SNe Ia.

\subsection{Conclusions}

We use the method of Han et al. (1994) to calculate the IFMR for stars of different metallicities. Our conclusions are as follows.

1. There is an obvious dependence of the IFMRs on the metallicity and the dependence is not monotone. When $Z=0.04$, the final mass of the CO WD for a given initial mass is smallest. For higher or lower $Z$, the mass of CO WD will be higher. The difference of the final mass derived from different metallicties is up to $0.4 M_{\odot}$.

2. The initial-final mass relationship for stars of $Z=0.02$ is consistent with observations.

3. For $Z \geq 0.02$, a helium white dwarf is formed from a star of $M_{\mathrm{i}} \leq 1.0 M_{\odot}$. The final masses of helium WDs for a given initial mass slightly decreases with metallicity.

4. Incorporating the IFMR for stars of $Z=0.02$ in the paper into Hurley's single stellar population synthesis code, we reproduce the mass distribution of DA WDs in Sloan DR4 except for some extra-massive WDs. 
5. We reconfirm the discovery of Kalirai et al. (2005) that the initial-final mass relationship derived from observations in NGC 2099 might be evidence of the dependence of the IFMR on metallicities and that the metallicity of NGC 2099 may be about 0.01, although the observational error of white dwarfs in NGC 2099 is large. More accurate observations have required to find a larger sample of white dwarfs in globular clusters. Such programs may help to confirm the dependence of the initial-final mass relationship on the metallicity.

6. We bring up again Willson's suggestion (Willson 2000) that the effect of metallicity may be the origin of the phenomenon that the mean mass of WDs decrease with effective temperature.

Acknowledgements. We thank Dr. Richard Pokorny for his kind help in improving this paper. This work was supported in part by the Natural Science Foundation of China under Grant Nos. 10433030, 10521001, 10603013 and 2007 CB815406 and Chinese Academy of Science under Grant No. O6YQ011001, Yunnan National Science Foundation (No. 2004A0022Q).

\section{Appendix A: Fitted formulae of initial-final mass relationship for stars of different metallicities}

The initial-final mass relationship for stars of different metallicities can be approximated by two parabola segments, except for the case of $Z=0.0001$. In this Appendix, we present the formulae fitted from our results for different metallicities. Hereafter, $M_{\mathrm{i}}$ is the initial mass of stars and $M_{\mathrm{f}}$ is the final mass of stars.

For $Z=0.1$,

$M_{\mathrm{f}}=\max \left[M_{\mathrm{f}, 1}, M_{\mathrm{f}, 2}\right], 1.0 M_{\odot}<M_{\mathrm{i}} \leq 5.5 M_{\odot}$, err $<1.0 \%,(\mathrm{~A} .1)$

where

$M_{\mathrm{f}, 1}=0.5675+0.1027 M_{\mathrm{i}}-0.0006261 M_{\mathrm{i}}^{2}$,

and

$M_{\mathrm{f}, 2}=0.7345-0.07362 M_{\mathrm{i}}+0.03973 M_{\mathrm{i}}^{2}$.

For $Z=0.08$,

$M_{\mathrm{f}}=\min \left[M_{\mathrm{f}, 1}, M_{\mathrm{f}, 2}\right], 1.0 M_{\odot} \leq M_{\mathrm{i}} \leq 6.0 M_{\odot}$, err $<1.8 \%,(\mathrm{~A} .4)$

where

$M_{\mathrm{f}, 1}=0.7097-0.1930 M_{\mathrm{i}}+0.08235 M_{\mathrm{i}}^{2}$,

and

$M_{\mathrm{f}, 2}=0.8316-0.1183 M_{\mathrm{i}}+0.03511 M_{\mathrm{i}}^{2}$.

For $Z=0.06$,

$M_{\mathrm{f}}=\min \left[M_{\mathrm{f}, 1}, M_{\mathrm{f}, 2}\right], 0.8 M_{\odot} \leq M_{\mathrm{i}} \leq 6.5 M_{\odot}$, err $<2.3 \%,(\mathrm{~A} .7)$

where

$M_{\mathrm{f}, 1}=0.5806-0.06852 M_{\mathrm{i}}+0.03928 M_{\mathrm{i}}^{2}$,

and

$M_{\mathrm{f}, 2}=0.8957-0.1313 M_{\mathrm{i}}+0.03004 M_{\mathrm{i}}^{2}$.

For $Z=0.05$,

$M_{\mathrm{f}}=\min \left[M_{\mathrm{f}, 1}, M_{\mathrm{f}, 2}\right], 0.8 M_{\odot} \leq M_{\mathrm{i}} \leq 6.5 M_{\odot}$, err $<1.8 \%,(\mathrm{~A} .10)$

where

$M_{\mathrm{f}, 1}=0.5736-0.06446 M_{\mathrm{i}}+0.03621 M_{\mathrm{i}}^{2}$, and

$M_{\mathrm{f}, 2}=0.8971-0.1255 M_{\mathrm{i}}+0.02750 M_{\mathrm{i}}^{2}$.

For $Z=0.04$,

$M_{\mathrm{f}}=\min \left[M_{\mathrm{f}, 1}, M_{\mathrm{f}, 2}\right], 0.8 M_{\odot} \leq M_{\mathrm{i}} \leq 6.5 M_{\odot}$, err $<1.9 \%$,

where

$M_{\mathrm{f}, 1}=0.5737-0.06207 M_{\mathrm{i}}+0.03353 M_{\mathrm{i}}^{2}$,

and

$M_{\mathrm{f}, 2}=0.8691-0.1107 M_{\mathrm{i}}+0.02491 M_{\mathrm{i}}^{2}$.

For $Z=0.03$,

$M_{\mathrm{f}}=\min \left[M_{\mathrm{f}, 1}, M_{\mathrm{f}, 2}\right], 0.8 M_{\odot} \leq M_{\mathrm{i}} \leq 6.5 M_{\odot}$, err $<2.3 \%,(\mathrm{~A} .16)$ where

$M_{\mathrm{f}, 1}=0.5701-0.05225 M_{\mathrm{i}}+0.03013 M_{\mathrm{i}}^{2}$,

and

$M_{\mathrm{f}, 2}=0.9897-0.1608 M_{\mathrm{i}}+0.03022 M_{\mathrm{i}}^{2}$.

For $Z=0.02$,

$M_{\mathrm{f}}=\min \left[M_{\mathrm{f}, 1}, M_{\mathrm{f}, 2}\right], 0.8 M_{\odot} \leq M_{\mathrm{i}} \leq 6.0 M_{\odot}$, err $<2.1 \%(\mathrm{~A} .19)$ where

$M_{\mathrm{f}, 1}=0.5716-0.04633 M_{\mathrm{i}}+0.02878 M_{\mathrm{i}}^{2}$,

and

$M_{\mathrm{f}, 2}=1.1533-0.2422 M_{\mathrm{i}}+0.04091 M_{\mathrm{i}}^{2}$.

For $Z=0.01$,

$M_{\mathrm{f}}=\min \left[M_{\mathrm{f}, 1}, M_{\mathrm{f}, 2}\right], 0.8 M_{\odot} \leq M_{\mathrm{i}} \leq 5.5 M_{\odot}$, err $<2.2 \%(\mathrm{~A} .22)$ where

$M_{\mathrm{f}, 1}=0.5897-0.05631 M_{\mathrm{i}}+0.03395 M_{\mathrm{i}}^{2}$,

and

$M_{\mathrm{f}, 2}=0.8660-0.1240 M_{\mathrm{i}}+0.03183 M_{\mathrm{i}}^{2}$.

For $Z=0.004$,

$M_{\mathrm{f}}=\min \left[M_{\mathrm{f}, 1}, M_{\mathrm{f}, 2}\right], 0.8 M_{\odot} \leq M_{\mathrm{i}} \leq 5.0 M_{\odot}$, err $<2.2 \%,(\mathrm{~A} .25)$ where

$M_{\mathrm{f}, 1}=0.5983-0.04908 M_{\mathrm{i}}+0.03780 M_{\mathrm{i}}^{2}$,

and

$M_{\mathrm{f}, 2}=0.8529-0.1056 M_{\mathrm{i}}+0.03194 M_{\mathrm{i}}^{2}$.

For $Z=0.001$,

$M_{\mathrm{f}}=\min \left[M_{\mathrm{f}, 1}, M_{\mathrm{f}, 2}\right], 0.8 M_{\odot} \leq M_{\mathrm{i}} \leq 4.5 M_{\odot}$, err $<1.0 \%,(\mathrm{~A} .28)$

where

$M_{\mathrm{f}, 1}=0.5959-0.007419 M_{\mathrm{i}}+0.03297 M_{\mathrm{i}}^{2}$,

and

$M_{\mathrm{f}, 2}=0.6312-0.04458 M_{\mathrm{i}}+0.04213 M_{\mathrm{i}}^{2}$.

For $Z=0.0003$,

$M_{\mathrm{f}}=\min \left[M_{\mathrm{f}, 1}, M_{\mathrm{f}, 2}\right], 1.0 M_{\odot} \leq M_{\mathrm{i}} \leq 4.5 M_{\odot}$, err $<0.4 \%,(\mathrm{~A} .31)$ where

$M_{\mathrm{f}, 1}=0.6300+0.007371 M_{\mathrm{i}}+0.02700 M_{\mathrm{i}}^{2}$,

and

$M_{\mathrm{f}, 2}=0.7629-0.03288 M_{\mathrm{i}}+0.02390 M_{\mathrm{i}}^{2}$.

For $Z=0.0001$,

$M_{\mathrm{f}}=0.6033+0.06839 M_{\mathrm{i}}+0.008401 M_{\mathrm{i}}^{2}$,

$1.0 M_{\odot} \leq M_{\mathrm{i}} \leq 4.5 M_{\odot}$, err $<1.0 \%$. 


\section{References}

Alexander, D. R., \& Ferguson, J. W. 1994, ApJ, 437, 879

Alcock, C., Allsman, R. A., \& Alves, D. 1999, ASPC, 165, 362

Arnett, W. D. 1982, ApJ, 253, 785

Busso, M., Gallino, R., \& Wasserburg, G. J. 1999, ARA\&A, 37, 239

Bedin, L. B., Salaris, M., Piotto, G., et al. 2005, ApJ, 624, L45

Blöcker, T. 1995, A\&A, 297, 727

Branch, D. 2004, Nature, 431, 1044

Branch, D., \& Bergh, S. V. 1993, AJ, 105, 2231

Buzzoni, A., Arnaboldi, M., \& Hodgkin, R. L. 2006, MNRAS, 368, 877

Cassisi, S., Castellani, V., Ciareclluti, P., et al. 2000, MNRAS, 315, 679

Cappellaro, E., Turatto, M., Tsvetkov, D. Y., et al. 1997, A\&A, 322,431

Chabrier, G. 1999, ApJ, 513, L103

Chaboyer, B., Green, E. M., \& Liebert, J. 1999, AJ, 117, 1360

Chen, X., \& Tout, C. A. 2007, ChJAA, 7, 2, 245

Claver, C. F., Liebert, J., Pergeron, P., et al. 2001, ApJ, 563, 987

Dobbie, P. D., Napiwotzki, R., Burleigh, M. R., et al. 2006b, MNRAS, 369, 383

Dominguez, I., Chieffi, A., Limongi, M., et al. 1999, ApJ, 524, 226

Eggleton, P. P. 1971, MNRAS, 151, 351

Eggleton, P. P. 1972, MNRAS, 156, 361

Eggleton, P. P. 1973, MNRAS, 163, 279

Eisenstein, D. J., Liebert, J., Harris, H. C., et al. 2006, ApJS, 167, 40

Ferrario, L., Wickramasinghe, D., Liebert, J., et al. 2005, MNRAS, 361, 1131

Finley, D., \& Koester, D. 1997, ApJ, 489, L79

Fontaine, G., Brassard, P., \& Bergeron, P. 2001, PASP, 113, 409

Forestini, M., \& Charbonnel, C. 1997, A\&AS, 123, 241

Gates, E., Gyuk, G., Harris, H. C., et al. 2004, ApJ, 612, L129

Gesicki, K., \& Zijlstra, A. A. 2007, A\&A, 467, L29

Girardi, L., Bressan, A., \& Bertelli, G. 2000, A\&A, 141, 371

Gratton, R., Bragaglia, A., Carretta, E., et al. 2006, ApJ, 642, 462

Groenewegen, M. A. T., \& de Jong, T. 1994, A\&A, 283, 463

Hachisu, I., \& Kato, M. 2003a, ApJ, 588, 1003

Hachisu, I., \& Kato, M. 2003b, ApJ, 590, 445

Hachisu, I., Kato, M., Nomoto, K., et al. 1999a, ApJ, 519, 314

Hachisu, I., Kato, M., \& Nomoto, K. 1999b, ApJ, 522, 487

Hamuy, M., Phillips, M. M., Schommer, R. A., et al. 1996, AJ, 112, 2391

Han, Z. 1998, MNRAS, 296, 1019

Han, Z., Podsiadlowski, P., \& Eggleton, P. P. 1994, MNRAS, 270, 121

Han, Z., Eggleton, P. P., Podsiadlowski, P., \& Tout, C. A. 1995, MNRAS, 277, 1443

Hansen, B. M. S. 2003, ApJ, 582, 915

Herwig, F. 1995, LIACo, 32, 441

Hillebrandt, W., \& Niemeyer, J. C. 2000, ARA\&A, 38, 191

Hurley, J. R., Pols, O. R., \& Tout, C. A. 2000, MNRAS, 315, 543

Hurley, J. R., Tout, C. A., \& Pols, O. R. 2002, MNRAS, 329, 897

Iben, I. 1983, ARA\&A, 21, 271

Iben, I., Jr., \& Tutukov, A. V. 1984, ApJS, 54, 335

Iglesias, C. A., \& Rogers, F. J. 1996, ApJ, 464, 943

Ihara, Y., Ozaki, J., Doi, M., et al. 2007, PASJ, 59, 811

Judge, P. G., \& Stencel, R. E. 1991, ApJ, 371, 357

Kalirai, J. S., Ventura, P., Richer, H. B., Fahlman, G. G., et al. 2001, AJ, 122, 3239

Kalirai, J. S., Richer, H. B., Reitzel, D., et al. 2005, ApJ, 618, L123

Kalirai, J. S., Bergeron, P., Hansen, B. M. S., et al. 2007a, ApJ, 671, 748

Kalirai, J. S., Hansen, B. M. S., Kelson, D. D., et al. 2007b [arXiv:0706.3894]

Kawka, A., Vennes, S., Oswalt, T. D., et al. 2006, ApJ, 643, L123

Kilic, M., Prieto, A., Brown, W. R., et al. 2007a, ApJ, 660, 1451

Kilic, M., Brown, W. R., Prieto, A., et al. 2007b, ApJ, 664, 1088

Kilic, M., Stanek, K. Z., \& Pinsonneault, M. H. 2007c, ApJ, 671, 761
Kippenhahn, R., \& Weigert, A. 1990, Stellar structure and evolution (Springer-Verlag), 102 or 374

Kepler, S. O., Kleinman, S. J., Nitta, A., et al. 2007, MNRAS, 375, 1315

Koester, D., \& Reimers, D. 1996, A\&A, 313, 810

Langer, N., Deutschmann, A., Wellstein, S., et al. 2000, A\&A, 362, 1046

Leibundgut, B. 2000, A\&ARv, 10, 179

Li, X. D., \& van den Heuvel, E. P. J. 1997, A\&A, 322, L9

Liebert, J., Bergeron, P., Eisenstein, D., et al. 2004, ApJ, 606, L147

Liebert, J., Bergeron, P., \& Holberg, J. B. 2005, ApJS, 156, 47

Luck, R. E., \& Heiter, U. 2007, AJ, 133, 2464

Marigo, P. 2001, A\&A, 370, 194

Mathews, W. G. 1990, ApJ, 354, 468

Meng, X., Chen, X., Tout, C. A., et al. 2006, ChJAA, 6, 4, 461

Miller, G. E., \& Scalo, J. M. 1979, ApJS, 41, 513

Moehler, S., Koester, D., Zoccali, M., et al. 2004, A\&A, 420, 515

Nomoto, K., Umeda, H., Hachisu, I., et al. 1999, in Type Ia Suppernova: Theory and Cosmology, ed. J. Truran, \& T. Niemeyer (New York: Cambridge Univ. Press), 63

Origlia, L., Valenti, E., Rich, R. M., et al. 2006, ApJ, 646, 499

Paczyński, B., \& Ziólkowski, L. 1968, Acta Astron., 18, 255

Perlmutter, S., Aldering, G., Goldhaber, G., et al. 1999, ApJ, 517, 565

Perryman, M. A. C., Brown, A. G. A., Lebreton, Y., et al. 1998, A\&A, 331, 81

Peterson, R. C., \& Green, E. M. 1998, ApJ, 502, L39

Phillips, M. M. 1993, ApJ, 413, L105

Podsiadlowski, P., Mazzali, P. A., Lesaffre, P., et al. 2006

[arXiv: astro-ph/0608324]

Pols, O. R., Tout, C. A., Eggleton, P. P., et al. 1995, MNRAS, 274, 964

Pols, O. R., Tout, C. A., Schröder, K. P., et al. 1997, MNRAS, 289, 869

Pols, O. R., Schröder, K. P., Hurly, J. R., et al. 1998, MNRAS, 298, 525

Prada Moroni, P. G., \& Straniero, O. 2002, ApJ, 581, 585

Prada Moroni, P. G., \& Straniero, O. 2007, A\&A, 466, 1043

Reid, I. N. 1996, AJ, 111, 2000

Reid, I. N., Turner, E. L., Turnbull, M. C., et al. 2007, ApJ, 665, 767

Reimers, D. 1975, Mem. R. Soc. liège, 6ième Serie, 8, 369

Riess, A. G., Filippenko, A. V., Challis, P., et al. 1998, AJ, 116, 1009

Roberts, M., \& Haynes, M. P. 1994, ARA\&A, 32, 115

Ruiz-Lapuente, P., Burkert, A., \& Canal, R. 1995, ApJ, 447, L69

Ruiz-Lapuente, P., Comeron, F., Mendez, J., et al. 2004, Nature, 431, 1069

Salpeter, E. E. 1955, ApJ, 121, 161

Schmidt, M. 1959, ApJ, 129, 243

Schröder, K. P., Pols, O. R., \& Eggleton, P. P. 1997, MNRAS, 285, 696

Shanks, T., Allen, P. D., Hoyle, F., et al. 2002, ASPC, 283, 274

Stetson, P. B., Bruntt, H., \& Grundahl, F. 2003, PASP, 115, 413

Straniero, O., Chieffi, A., Limongi, M., et al. 1997, ApJ, 478, 332

Terlevich, A. I., \& Forbes, D. A. 2002, MNRAS, 330, 547

Timmes, F. X., Diehl, R., \& Hartmann, D. H. 1997, ApJ, 479, 760

Timmes, F. X., Brown, E. F., \& Truran, J. W. 2003, ApJ, 590, L83

Travaglio, C., Hillebrandt, W., \& Reinecke, M. 2005, A\&A, 443, 1007

Umeda, H., Nomoto, K., Yamaoka, H., et al. 1999, ApJ, 513, 861

van Kerkwijk, M. H., Bassa, C. G., Jacoby, B. A., et al. 2005, ASPC, 328, 357

Valenti, J. A., \& Fischer, D. A. 2005, ApJS, 159, 141

Yungelson, L., Livio, M., Tutukov, A., et al. 1995, ApJ, 447, 656

Wang, L., Höflich, P., \& Wheeler, J. C. 1997, ApJ, 483, L29

Webbink, R. F. 1984, ApJ, 277, 355

Whelan, J., \& Iben, I. 1973, ApJ, 186, 1007

Weidemann, V. 1977, A\&A, 59, 411

Weidemann, V. 2000, A\&A, 363, 647

Weidemann, V., \& Koester, D. 1983, A\&A, 121, 77

Williams, K. A. 2006 [arXiv: astro-ph/0610254]

Williams, K. A., Bolte, M., \& Koester, D. 2004, ApJ, 615, L49

Willson, L. A. 2000, ARA\&A, 38, 573 